\title{
Mapping urban impervious surfaces from an airborne hyperspectral imagery using the object- oriented classification approach
}

\author{
Rahim Aguejdad ${ }^{1, *}$, Aziz Serradj ${ }^{1}$, and Christiane Weber $^{2}$ \\ ${ }^{1}$ Laboratoire Image Ville Environnement UMR7362-CNRS, 3 Rue de l'Argonne, 67000 Strasbourg, \\ France \\ ${ }^{2}$ Maison de la Télédétection UMR TETIS, 500 Rue Jean-François Breton, 34000 Montpellier, \\ France
}

\begin{abstract}
The objective of this research is to explore the capabilities of the hyperspectral imagery in mapping the urban impervious objects and identifying the surface materials using an object-oriented approach. The application is conducted to Toulouse city (France) within the HYEP research project in charge of using hyperspectral imagery for the environmental urban planning. The method uses the multi-resolution segmentation and classification algorithms. The first results highlight a high potential of the hyperspectral imagery in land cover mapping of the urban environment, especially the extraction of impervious surfaces. They, also, illustrate, that the object-oriented approach by means of the fuzzy logic classifier yields promising results in distinguishing the mean roofing materials based only on the spectral information. Conversely to the red clay tiles and metal roofs, which are easily identified, the concrete, gravel and asphalt roofs are still confused with roads.
\end{abstract}

\section{Introduction}

Remote sensing technology has a great potential to afford consistent and useful spatial information at different spatial and temporal scales. It can help understanding the links between land use and infrastructure change and a variety of social, economic, demographic and environmental processes $[1,2]$. This information derived from remotely sensed datasets leads to an improved observation and monitoring that can benefit applied urban planning and management [2]. However, land cover mapping of the urban environment by means of the remote sensing remains a distinct challenge due to the strong diversity of materials and objects, leading to spectral heterogeneity and geometric complexity of the urban areas as well as the presence of the shadow scenes [3]. In fact, several studies have showed that current satellite data provide limited information to deal with the richness, heterogeneity and complexity of the materials and forms in the urban areas. For instance, [4] has showed the limits of the multispectral sensors for the urban impervious surfaces extraction compared to

\footnotetext{
* Corresponding author: rahim.aguejdad@live-cnrs.unistra.fr
} 
the hyperspectral imagery capabilities. In addition to that, intra-urban discrimination of land cover and land use types is needed for many local scales remote sensing applications requirements [2]. Accordingly, several studies have shown that a spatial resolution of at least $5 \mathrm{~m}$ is necessary to accurately extract the land cover objects $[5,6]$. In addition to the spatial accuracy of the sensor, the definition of the thematic mapping classes and the image classification accuracy require specific spectral characteristics ranging from the Visible Near-Infrared (VNIR) to Short Wave Infrared (SWIR) wavelength domains [7, 8].

The recent introduction of advanced high spatial resolution hyperspectral sensors provides new perspectives [9]. Thanks to its high spectral capacities in identifying different urban objects [10], the hyperspectral imagery is used to address key science and application questions in a wide array of fields, ranging from ecosystem function and diversity to human health, precision agriculture and urbanization. For example, [11] have used hyperspectral aerial images for mapping asbestos cement roofs and developed a spectral index related to the roof weathering status. At the same time, the recent developments in hyperspectral domain have stressed on the need for advanced classification methods [12]. Recently, [13] proposed a generic method for fusion of hyperspectral and VHR multispectral image classifications in urban areas. The proposed fusion model relies on posterior class probabilities which are integrated through an energy minimization process [13]. Furthermore, [12] have proposed a new methodology for spectral-spatial classification of hyperspectral images. This method, which is as an alternative to current pixel-wise methods, simultaneously considers the spectral and spatial information [12].

The hyperspectral users' community in France has addressed very detailed science needs for a high-resolution hyperspectral mission in the following themes: the study of vegetation, the coastal and inland water ecosystems, geosciences, the urban environment, the atmospheric studies, the security and defence [14]. Accordingly, the HYPXIM task specification work has been conducted to define a hyperspectral camera with high spatial resolution $(8 \mathrm{~m})$ coupled to a panchromatic camera with $2 \mathrm{~m}$ spatial resolution. To simulate the images acquired by such space instrument, airborne acquisition missions were carried out. The objective is to assess the gain provided by the HYPXIM mission compared to existing missions such as the multispectral or hyperspectral Pleiades coming from EnMAP (30 $\mathrm{m})$ and Prisma $(20 \mathrm{~m})$.

The aim of this paper is to analyze the potential of the simulated datasets provided by the airborne hyperspectral imagery in mapping urban impervious surfaces using an objectoriented classification approach compared to an analysis on per-pixel basis. The study is applied to Toulouse city (France) within the HYEP (Hyperspectral imagerY for Environmental urban Planning) research project in charge of using the hyperspectral imagery for the environmental urban planning.

\section{Materials and Methods}

\subsection{Study area and data}

The study area is the city of Toulouse $\left(43^{\circ} 36^{\prime} 19^{\prime \prime} \mathrm{N}, 1^{\circ} 26^{\prime} 34^{\prime \prime} \mathrm{E}\right)$ which is located in the South-Western of France (Fig. 1). The used hyperspectral imagery is derived from the simulation of the future HYPXIM sensor using data acquired by the HySpex hyperspectral camera (www.hyspex.no) over the city centre in 2015. It records data with a high number of narrowly defined spectral bands (192). It provides a continuous spectrum ranging from 400 $\mathrm{nm}$ to $2500 \mathrm{~nm}$, covering both VNIR as well as SWIR domains (Table 1) with $4 \mathrm{~m}$ ground geometric resolution. 


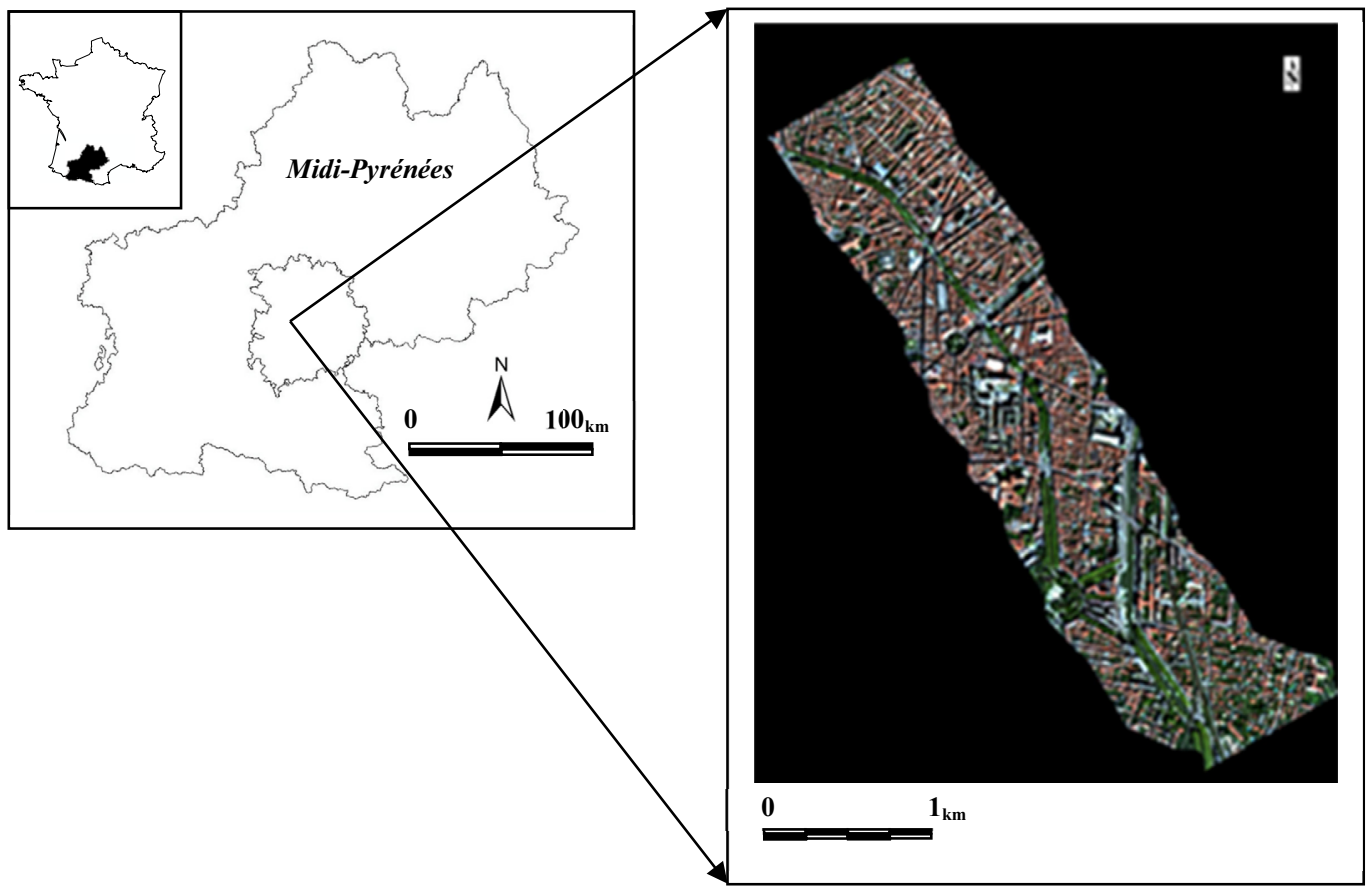

Fig. 1. RGB hyperspectral image centred over the dense city centre of Toulouse (Toulouse ONERA, 2015).

The HySpex hyperspectral VNIR-SWIR georeferenced radiances image $(0.4 \mu \mathrm{m}-2.5 \mu \mathrm{m}$; $\mathrm{GSD}=2 \mathrm{~m}$ ) is used to simulate different spatial and spectral resolutions, corresponding to the HYPXIM sensor which is under study and the operative PLEIADES and SENTINEL-2 instruments. The HYPXIM sensor is the future hyperspectral space borne instrument leaded by the CNES (Centre National d'Etudes Spatiales). It is expected to provide data with a spatial resolution of $8 \mathrm{~m}$ in the VNIR-SWIR range.

Table 1. Hyperspectral data spectral characteristics.

\begin{tabular}{|c|c|c|c|}
\hline Domain & $\begin{array}{c}\text { Spectrum } \\
(\mathbf{n m})\end{array}$ & $\begin{array}{c}\text { Band width } \\
\boldsymbol{\Delta} \boldsymbol{\lambda}(\mathbf{n m})\end{array}$ & Bands list \\
\hline Visible (Vis) & $418-691$ & 10.9 & $1-26$ \\
\hline Near-InfraRed (NIR) & $702-1095$ & 10.9 & $27-63$ \\
\hline Short Wave InfraRed (SWIR) & $1105-2502$ & 10.9 & $64-192$ \\
\hline
\end{tabular}

The BDTOPO $^{\circledR}$ topographic database will be used as a reference map to validate the segmentation and classification results. The BDTOPO ${ }^{\circledR}$ is a $3 \mathrm{D}$ topographic database that provides a vector and structured description of the spatial objects with a metric mapping accuracy designed to scales ranging from $1 / 5000$ to $1 / 50000$. The segmentation accuracy will be assessed by comparing the created image objects with the building blocks from the BDTOPO $^{\circledR}$. The validation feedbacks will allow setting the adequate input segmentation parameters and then refining the delineation of the borders of objects. 


\subsection{Method}

An image based supervised classification is performed using an object-oriented approach by means of the eCognition software. First, a routine image pre-processing including radiometric, atmospheric, and geometric corrections is performed. The atmospheric correction is carried out using the COCHISE (atmospheric correction code for hyperspectral images of remote-sensing sensors) algorithm [15]. Second, the image segmentation is performed using all the spectral bands except those presenting an excessively noise. A multiresolution segmentation algorithm is performed to create meaningful image objects using only the spectral information. The scale factor is set to 0.4 , whereas the shape criterion is not taken into consideration. These segmentation parameters are derived based on several tests, which are performed to deal with the characteristics of the target objects present in the study area. Then, spectral difference segmentation is applied to merge spectrally similar neighboring image objects if the difference between their layer mean intensities is below the user-defined threshold value given by the maximum spectral difference. The last step consists in classifying the image objects with respect to a predefined hierarchical network. The supervised classification procedure uses the fuzzy logic classifier which is based on the use of membership functions.

\section{Results and discussion}

Fig. 2 shows the image objects as the output of the segmentation process. The highly heterogeneous patterns of development (residential vs commercial, individual vs collective housing, low and high density, geometry, roof materials...) limits the application of the same segmentation parameters. In addition to that, the presence of "mixed" pixels that compose the borders of objects leads to their inaccurate delineation. One of the solutions is to apply the process of unmixing. Another alternative consists in using different input segmentation criteria after splitting the study area into homogeneous zones.

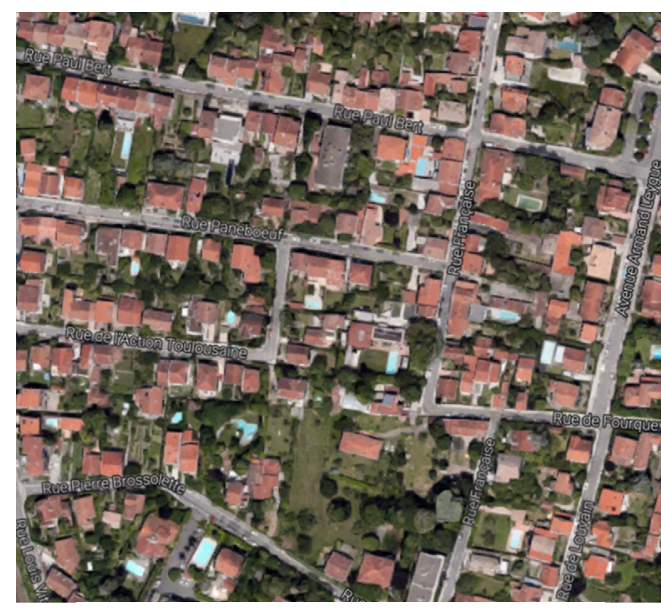

Image from Google Maps@ 2017

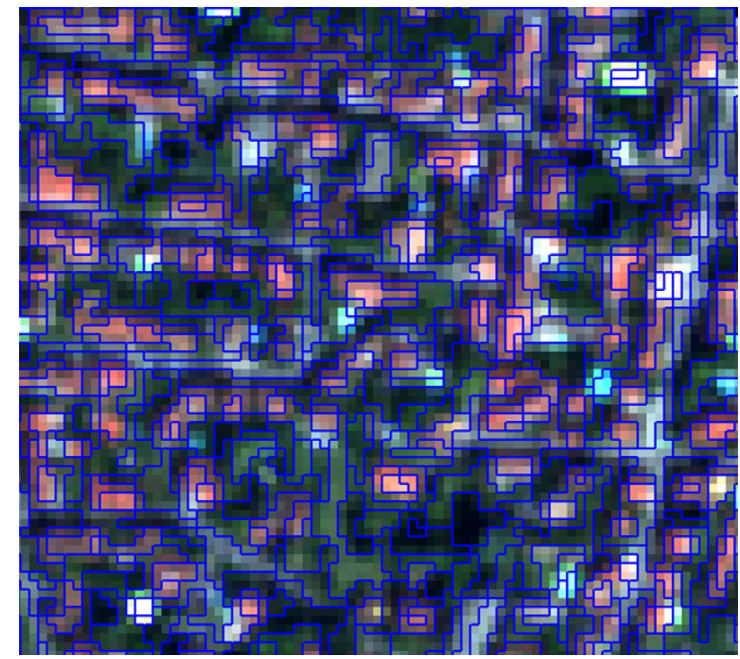

$100 \mathrm{~m}$

Fig. 2. Image objects in a residential area of low density and highly dominated by individual houses. 
Fig. 3 shows the map of the buildings that are extracted by means of the classification of the image objects. It illustrates that the classification algorithm performs in mapping red clay tiles and metal building' roofs. These well-classified segments consist in bright construction materials (Zinc, Wood, PVC).

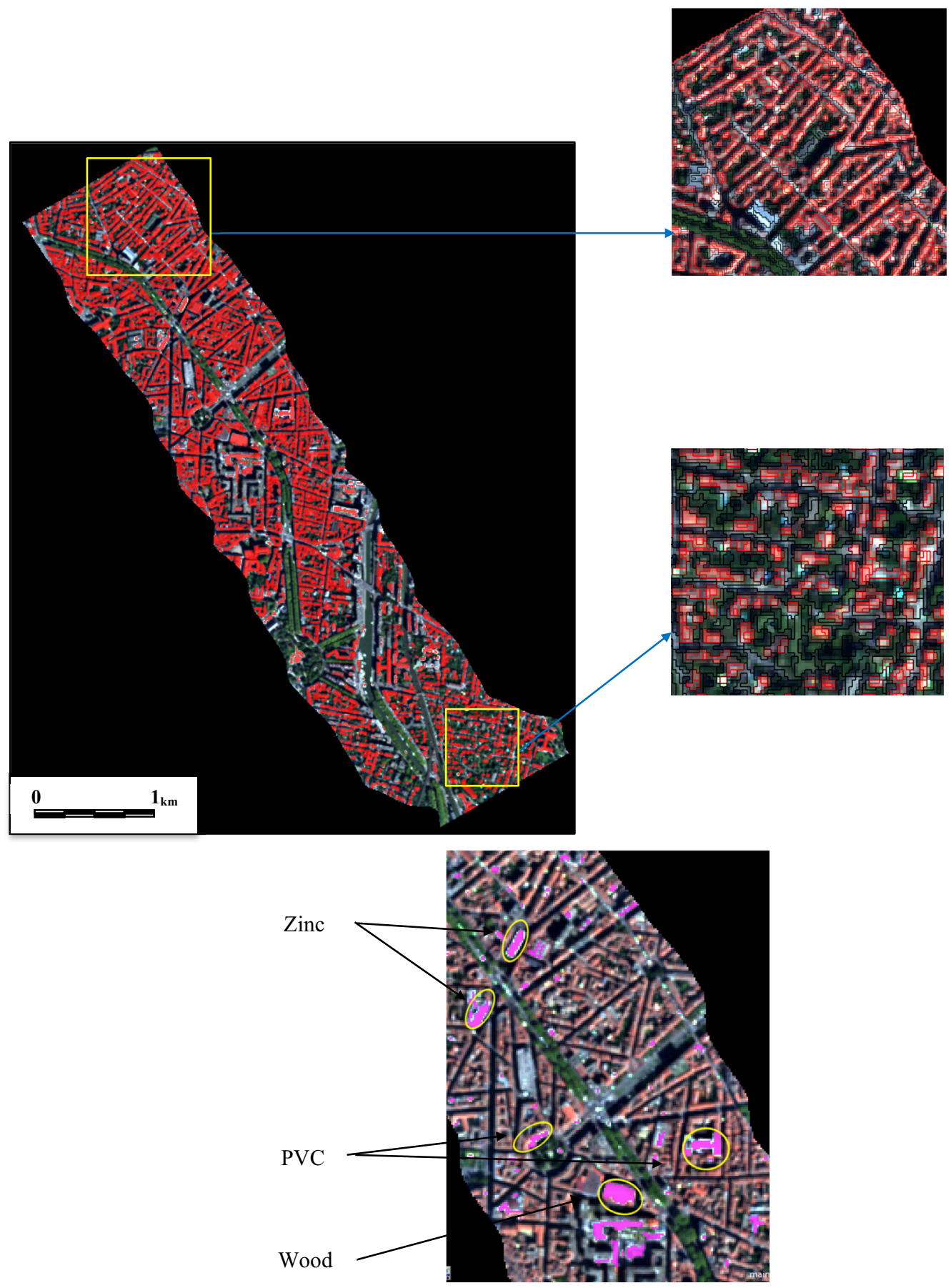

Fig. 3. Extraction of red clay tiles and metal roofs buildings. 
Despite the obvious merits that the used hyperspectral imagery may offer based on the 192 provided spectral bands, great challenges need to be addressed such as the observed confusion between the spectral signatures similarity between some urban land use and cover objects. For instance, buildings with concrete, gravel or bitumen roofs present similar reflectance behavior compared to the road network and associated structures such as car parks (Fig. 4).

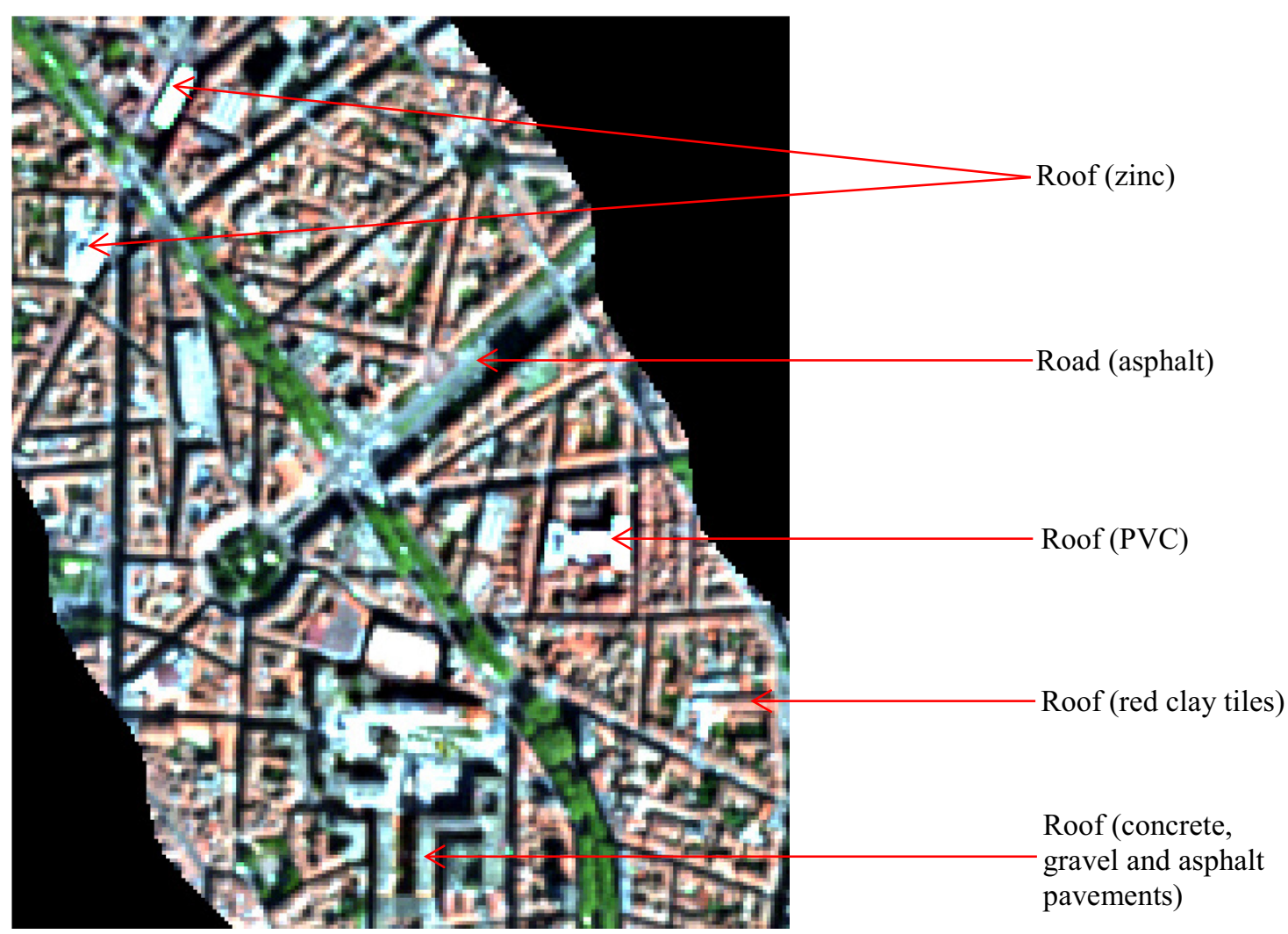

Fig. 4. Spectral similarity problem between roads and asphalt or concrete grey pavements roofs.

Fig. 5 illustrates the extent to which the reflectance curves of roads and grey roofs over the VNIR and SWIR wavelength spectral domains are close to each other.

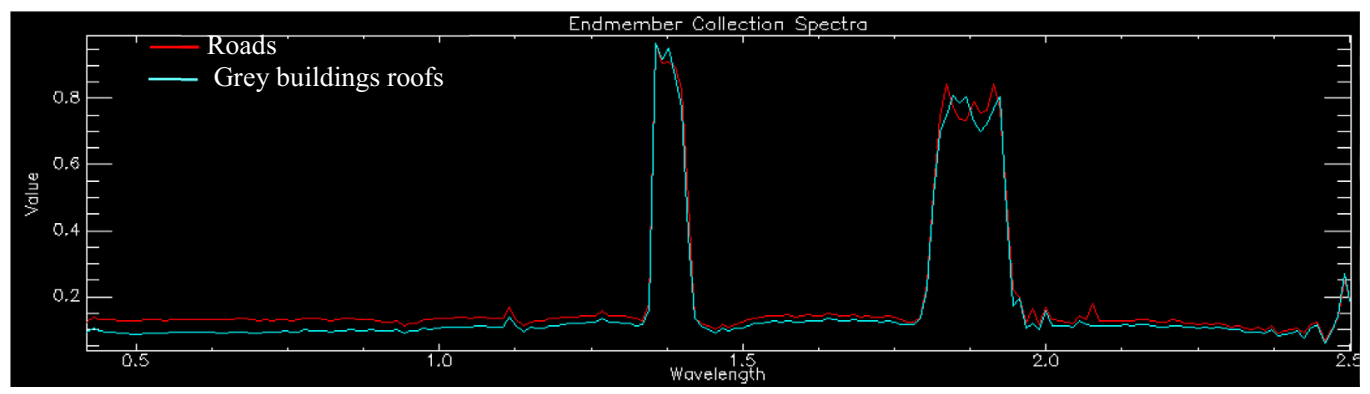

Fig. 5. Spectral profiles similarity between roads and dark roofs. 
In addition to the spectral information, one solution consists in the use of complementary information (3D information, texture, morphology and contextual measurements) that will probably help to improve the fuzzy classification outputs. The use of the elevation information such as LiDAR data as a thematic layer in combination with a hyperspectral imagery in the classification process will allow to deal with the omnipresence of confusion between roads and concrete, gravel or bitumen buildings roofs.

\section{Conclusion}

This study highlights the high potential of the simulated HYPXIM hyperspectral imagery for the urban land cover mapping, especially the extraction of impervious surfaces. It, also, illustrates that the object-oriented classification approach by means of the fuzzy logic method yields promising results in identifying the mean construction roof' materials based on the spectral information, only. Indeed, the red clay tiles and metal roofs are easily distinguished compared to the grey roofs that are frequently confused with the roads. One of the future perspectives is to compare the object-oriented method to the Super Angular Mapper (SAM) classifier that will be trained through a set of samples (roads, vegetation, water, and buildings) selected from an external spectral library. The comparison and accuracy assessment of the two methods will be based on quantitative measurements such as the Kappa statistic using the BDTOPO ${ }^{\circledR}$ as the reference map. Besides, this work, which is in progress, will be applied to different spatial resolutions datasets $(2,4,8$ and $10 \mathrm{~m})$.

This research is supported by the French ANR HYEP (Hyperspectral imager Y for Environmental urban Planning) project. The authors would like to thank the anonymous reviewer for his comments and suggestions that helped us to significantly improve this paper.

\section{References}

1. R.R. Rindfuss, P.C. Stern, Linking remote sensing and social science. In S. Liverman et al. (Eds.), People and pixel-linking remote sensing and social science (pp. 1-28). Washington, DC: National Academic Press (1998)

2. M. Herold, H. Couclelis, K.C. Clarke, The Role of Spatial Metrics in the Analysis and Modeling of Urban Land Use Change. Computers, Environment and Urban Systems 29, 369-399 (2005)

3. F. Priem, F. Canter, Synergistic Use of LiDAR and APEX Hyperspectral Data for High-Resolution Urban Land Cover Mapping. Remote sensing 8, 787 (2016)

4. D.S. Lu, Q.H. Weng, Use of impervious surface in urban land-use classification. Remote Sensing of Environment 102, 146-160 (2006)

5. R. Welch, Spatial resolution requirements for urban studies. International Journal of Remote Sensing 3(2), 139-146 (1982)

6. R.R. Jensen, P.J. Hardin, A.J. Hardin, Classification of urban tree species using hyperspectral data. Geocarto International 27, 443-458 (2012)

7. E. Ben-Dor, Imaging spectrometry for urban applications. In Imaging Spectrometry: Basic Principles and Prospective Applications; Van Der Meer, F.D., De Jong, S.M., Eds.; Kluwer Academic Publishers: Dordrecht, The Netherland; pp. 243-281 (2001)

8. D.A. Roberts, M. Herold, Imaging spectroscopy of urban materials. In Infrared Spectroscopy in Geochemistry, Exploration and Remote Sensing; King, P., Ramsey, M.S., Swayze, G., Eds.; Mineral Association of Canada: Ottawa, ON, Canada; pp. 155-181 (2004) 
9. S. Gadal, W. Ouerghemmi, Morpho-spectral objects classification by hyperspectral airborne imagery, $8^{\text {th }}$ Workshop in Hyperspectral Image and Signal Processing: Evolution in Remote Sensing, Aug. 2016, LosAngeles, USA (2016)

10. S. Pascucci, C. Bassani, R.M. Cavalli, L. Fusilli, A. Palombo, S. Pignatti, F. Santini, Hyperspectral remote sensing capability for mapping near-surface asbestos deposits and pollutants dispersion in soils. Proc. Hyperspectral 2010 Workshop, Frascati, Italy, 17-19 March (2010)

11. C. Cilia, C. Panigada, M. Rossini, G. Candiani, M. Pepe, R.Colombo, Mapping of asbestos cement roofs and their weathering status using hyperspectral aerial images. ISPRS International Journal of Geo-Information 4, 928-941(2015)

12. Z. Miao, W. Shi, A new methodology for spectral-spatial classification of hyperspectral images. Journal of Sensors, Vol. 2016, 12 pages (2016)

13. A. Hervieu, A. Le Bris, C. Mallet, Fusion of hyperspectral and VHR multispectral image classifications in urban areas. ISPRS Annals of Photogrammetry, Remote Sensing and the Spatial Information Sciences, Vol. III-3, Prague, Czech Republic, 12-19 July (2016)

14. S. Michel, M.J. Lefevre-Fonollosa, S. Hosford, HYPXIM-A hyperspectral satellite defined for science, security and defence users. Proc. 'Hyperspectral 2010 Workshop', Frascati, Italy, 17-19 March (2010)

15. Y. Boucher, L. Poutier, V. Achard, X. Lenot, C. Miesch, Validation and robustness of an atmospheric correction algorithm for hyperspectral images. Algorithms and Technologies for Multispectral, Hyperspectral, and Ultraspectral Imagery VIII, $\mathrm{N}^{\circ}$ 4725-54, SPIE AeroSense 2002, Orlando, FL., 1-5 April (2002) 